BMJ Open

Diabetes

Research

\& Care

\section{Renal hyperfiltration is independently associated with increased all-cause mortality in individuals with type 2 diabetes: a prospective cohort study}

To cite: Penno G, Orsi E, Solini A, et al. Renal hyperfiltration is independently associated with increased allcause mortality in individuals with type 2 diabetes: a prospective cohort study. BMJ Open Diab Res Care 2020;8:e001481. doi:10.1136/ bmjdrc-2020-001481

- Additional material is published online only. To view please visit the journal online (http://dx.doi.org/10.1136/ bmjdrc-2020-001481).

Received 19 April 2020 Revised 28 May 2020 Accepted 12 June 2020

Check for updates

(c) Author(s) (or their employer(s)) 2020. Re-use permitted under CC BY-NC. No commercial re-use. See rights and permissions. Published by BMJ.

For numbered affiliations see end of article.

Correspondence to

Professor Giuseppe Pugliese; giuseppe.pugliese@uniroma1.it

\section{ABSTRACT}

Introduction In addition to favoring renal disease progression, renal 'hyperfiltration' has been associated with an increased risk of death, though it is unclear whether and how excess mortality is related to increased renal function. We investigated whether renal hyperfiltration is an independent predictor of death in patients with type 2 diabetes from the Renal Insufficiency And Cardiovascular Events Italian multicenter study. Research design and methods This observational, prospective cohort study enrolled 15773 patients with type 2 diabetes consecutively attending 19 Italian diabetes clinics in 2006-2008. Serum creatinine, albuminuria, cardiovascular risk factors, and complications/ comorbidities were assessed at baseline. Vital status on 31 October 2015 was retrieved for 15656 patients (99.26\%). Patients were stratified $(A)$ by absolute estimated glomerular filtration rate (eGFR) values in eGFR deciles or Kidney Disease: Improving Global Outcomes (KDIGO) categories and (B) based on age-corrected thresholds or age and gender-specific 95th and 5th percentiles in hyperfiltration, hypofiltration, and normofiltration groups. Results The highest eGFR decile/category and the hyperfiltration group included (partly) different individuals with similar clinical features. Age and gender-adjusted death rates were significantly higher in deciles 1,9 , and $10\left(\geq 103.9,50.9-62.7\right.$, and $<50.9 \mathrm{~mL} / \mathrm{min} / 1.73 \mathrm{~m}^{2}$, respectively) versus the reference decile 3 (92.9-97.5 $\mathrm{mL} / \mathrm{min} / 1.73 \mathrm{~m}^{2}$ ). Mortality risk, adjusted for multiple confounders, was also increased in deciles 1 (HR 1.461 (95\% Cl 1.175 to 1.818$), p=0.001), 9(1.312(95 \% \mathrm{Cl}$ 1.107 to 1.555$), p=0.002)$, and $10(1.976$ (95\% Cl 1.673 to 2.333), $p<0.0001$ ) versus decile 3 . Similar results were obtained by stratifying patients by KDIGO categories. Death rates and adjusted mortality risks were significantly higher in hyperfiltering and particularly hypofiltering versus normofiltering individuals.

Conclusions In type 2 diabetes, both high-normal eGFR and hyperfiltration are associated with an increased risk of death from any cause, independent of confounders that may directly impact on mortality and/or affect GFR estimation. Further studies are required to clarify the nature of this relationship.

Trial registration number NCT00715481.

\section{Significance of this study}

What is already known about this subject?

- Previous reports have shown that, in addition to favoring renal disease progression, renal 'hyperfiltration' is associated with increased mortality.

- However, it is unclear whether (and how) the increased risk of death is related to increased renal function or to confounders that may directly impact on mortality and/or affect assessment of estimated glomerular filtration rate (eGFR).

What are the new findings?

- This large, observational, prospective cohort study showed that the highest eGFR decile/category (above $105 \mathrm{~mL} / \mathrm{min} / 1.73 \mathrm{~m}^{2}$ ) and the age (and gender-) corrected hyperfiltration groups included partly different individuals, though with similar clinical features (ie, younger age, more frequently male and current smoker, more favorable cardiovascular risk profile, lower prevalence of comorbidities).

- In addition to low eGFR/hypofiltration, both highnormal eGFR and hyperfiltration were associated with an increased risk of death from any cause.

- Increased mortality risk was independent of confounders that may directly impact on mortality and/ or affect GFR estimation.

How might these results change the focus of research or clinical practice?

- In individuals with type 2 diabetes, not only reduced, elevated eGFR, either in the high-normal or in the hyperfiltration range, should be considered a risk factor for increased mortality.

- Further studies are required to establish whether the increased mortality risk is directly related to eGFR and to clarify the mechanisms underlying this relationship.

\section{INTRODUCTION}

Renal (ie, whole-kidney) hyperfiltration is commonly defined as a glomerular filtration 
rate (GFR) that exceeds 2 SD above mean GFR in healthy individuals, ${ }^{1}$ corresponding to values ranging from 130 to $140 \mathrm{~mL} / \mathrm{min} / 1.73 \mathrm{~m}^{2}{ }^{2}$ An elevated GFR has been reported in the early stage of both type 1 and type 2 diabetes, with prevalence values of $10 \%-67 \%$ and $6 \%-73 \%^{3}$ and average increases of approximately $27 \%{ }^{4}$ and $16 \%,{ }^{5}$ respectively. Hyperfiltration has been reported also in non-diabetic obese individuals, though in most, but not all studies it was no longer observed when GFR was adjusted for body size. ${ }^{3}$ However, this definition of renal hyperfiltration does not consider a number of factors that impact on GFR variability, such as nephron endowment at birth, ethnicity, gender, and especially age. ${ }^{3}$ Indeed, after the age of 40 years, the threshold for hyperfiltration should be corrected for the annual GFR decline of $1 \mathrm{~mL} / \mathrm{min} / 1.73 \mathrm{~m}^{2}{ }^{6}$ Moreover, identification of hyperfiltration is complicated by the inaccuracy of serum creatinine-based GFR-estimating equations, ${ }^{3}$ which systematically underestimate GFR, especially in the normal or increased range, in individuals with type 2 diabetes. $^{7}$

Renal hyperfiltration occurring in early diabetes and obesity is the result of an increased single-nephron GFR (SNGFR), the so-called glomerular hyperfiltration, which is caused by multiple mechanisms affecting the determinants of glomerular ultrafiltration. ${ }^{8}$ Increased SNGFR may also occur despite normal or reduced whole-kidney GFR, as it represents an adaptive response for maintaining renal function in several renal disorders. A supraphysiological GFR elevation is considered a risk factor for adverse renal outcomes in diabetic individuals. ${ }^{3}$ Though not consistently, epidemiological surveys have in fact reported an association with subsequent GFR loss and development of microalbuminuria or macroalbuminuria in patients with type 1 and 2 diabetes. ${ }^{3}$ Likewise, under conditions of reduced renal mass, hyperfiltration in the residual nephrons is considered the main mechanism leading to further nephron loss and progression toward end-stage renal disease. ${ }^{9}$

In addition to favoring renal disease progression, renal hyperfiltration has been associated with increased mortality. A meta-analysis of general population and highrisk cohorts has shown an increased risk of death from any cause, but not from cardiovascular disease (CVD), in individuals with an estimated GFR (eGFR) $\geq 105 \mathrm{~mL} /$ $\mathrm{min} / 1.73 \mathrm{~m}^{2}$ and normoalbuminuria, ${ }^{10}$ a finding subsequently confirmed in people with and without diabetes or hypertension. ${ }^{11}{ }^{12}$ However, this increased mortality risk has been interpreted as an effect of conditions such older age and severe comorbidities, which are characterized by low creatinine values due to reduced muscle mass rather than to increased renal function. ${ }^{10}{ }^{12}$ A recent systematic review has suggested that renal hyperfiltration is indeed associated with a higher risk of death and CVD. ${ }^{13}$ Unfortunately, the studies considered in this review were mostly retrospective and included individuals from either the general population or selected disease groups of various size and different risk, and only some of them reported measurements of albuminuria/proteinuria or were specifically focused on hyperfiltration. More importantly, most of these studies did not evaluate 'hyperfiltering' individuals, but only those falling in the highest eGFR category, which included people with high-normal eGFR values below the hyperfiltration threshold, at variance with older patients falling in other categories, who may have exceeded the age-corrected threshold.

The present analysis aimed at assessing whether renal hyperfiltration at baseline, defined as either the highest eGFR decile/category or age (and gender-) corrected hyperfiltration, is an independent predictor of subsequent death from any cause in the large cohort of individuals with type 2 diabetes from the Renal Insufficiency And Cardiovascular Events (RIACE) Italian multicenter study.

\section{METHODS}

\section{Design}

The RIACE is an observational, prospective cohort study on the impact of eGFR on morbidity and mortality in individuals with type 2 diabetes. ${ }^{14}$

\section{Patients}

The study population included 15773 Caucasian patients (after excluding 160 individuals with missing or implausible values), consecutively attending 19 hospital-based, tertiary referral diabetes clinics of the National Health Service throughout Italy in 2006-2008. Exclusion criteria were dialysis or renal transplantation.

\section{All-cause mortality}

The vital status of study participants on 31 October 2015 was verified by interrogating the Italian Health Card database (http://sistemats1.sanita.finanze.it/wps/portal/), which provides updated and reliable information on all current Italian residents. $^{15}$

\section{Baseline measurements}

Baseline data were collected using a standardized protocol across participating centers. ${ }^{14}$

Participants underwent a structured interview in order to collect the following information: age, smoking status, known diabetes duration, comorbidities, and current glucose, lipid, and blood pressure (BP)-lowering treatments.

Body mass index (BMI) was calculated from weight and height, whereas waist circumference was estimated from log-transformed BMI values ${ }^{16}$; $\mathrm{BP}$ was measured with a sphygmomanometer with the patients seated with the arm at the heart level.

Hemoglobin A1c (HbAlc) was measured by highperformance liquid chromatography using Diabetes Control and Complications Trials-aligned methods; triglycerides and total and high-density lipoprotein (HDL) cholesterol were determined in fasting blood samples by colorimetric enzymatic methods; low-density 
lipoprotein (LDL) cholesterol was calculated by the Friedewald formula.

The presence of diabetic kidney disease (DKD) was assessed by measuring albuminuria and serum creatinine, as previously detailed. ${ }^{14}{ }^{17}$ Albumin excretion rate was obtained from 24 hours of urine collections or calculated from albumin-to-creatinine ratio in earlymorning, first-voided urine samples, using a conversion formula developed in patients with type 1 diabetes and preliminarily validated in a subgroup of RIACE participants. Albuminuria was measured in fresh urine samples by immunonephelometry or immunoturbidimetry, in the absence of interfering clinical conditions. One to three measurements for each patient were obtained; in cases of multiple measurements, the geometric mean of 2-3 values was used for analysis. In individuals with multiple measurements, the concordance rate between the first value and the geometric mean was $>90 \%$ for all albuminuria categories. ${ }^{17}$ Serum (and urine) creatinine was measured by the modified Jaffe method, traceable to Isotope-Dilution Mass Spectrometry, and eGFR was calculated by the Chronic Kidney Disease Epidemiology Collaboration equation. ${ }^{15}$

In each center, the presence of diabetic retinopathy (DR) was assessed by an expert ophthalmologist by dilated funduscopy. Patients with mild or moderate nonproliferative DR were classified as having non-advanced DR, whereas those with severe non-proliferative DR, proliferative DR, or maculopathy were grouped into the advanced DR category. DR grade was assigned based on the worse eye. ${ }^{18}$

Previous major acute CVD events, including myocardial infarction, stroke, foot ulcer/gangrene/amputation, and coronary, carotid, and lower limb revascularization, were adjudicated based on hospital discharge records by an ad hoc committee in each center. ${ }^{19}$

\section{Categorization of patients}

For the purpose of the current analysis, the RIACE participants were either stratified by absolute eGFR levels or categorized based on age (and gender-) adjusted eGFR threshold for hyperfiltration (and hypofiltration), as shown in online supplementary table S1. First, the cohort was divided into deciles of baseline eGFR values, from the highest $\left(>104 \mathrm{~mL} / \mathrm{min} / 1.73 \mathrm{~m}^{2}\right)$ to the lowest $(<51 \mathrm{~mL} /$ $\min / 1.73 \mathrm{~m}^{2}$ ). As the highest decile approximately corresponds to the high-normal G1 subcategory, patients were also stratified according to the following Kidney Disease: Improving Global Outcomes (KDIGO) eGFR categories and subcategories $\left(\mathrm{mL} / \mathrm{min} / 1.73 \mathrm{~m}^{2}\right)$ : Gla $(\geq 105)$, G1b (90-104), G2 (60-89), G3a (45-59), G3b (30-44), G4-5 $(<30) .{ }^{20}$ Then, participants were classified as hyperfiltering if their baseline eGFR value was above an ageadjusted hyperfiltration threshold calculated according to the formula $130 \mathrm{~mL} / \mathrm{min} / 1.73 \mathrm{~m}^{2}-1.0 \mathrm{~mL} / \mathrm{min} / 1.73$ $\mathrm{m}^{2}$ per year after 40 years of age. ${ }^{21}$ The remaining individuals were classified as normofiltering or hypofiltering based on whether their baseline eGFR value was above or below $60 \mathrm{~mL} / \mathrm{min} / 1.73 \mathrm{~m}^{2}$. According to previous studies, ${ }^{22}{ }^{23}$ hyperfiltering and hypofiltering patients were also defined as individuals with eGFR values above the 95th and below the 5th age and gender-specific percentiles, respectively, whereas normofiltering individuals were those falling in between. As shown in online supplementary figure S1, both percentiles were virtually identical in men and women; in addition, while there was a high concordance between the two definitions for hyperfiltration, the 5th percentile threshold for hypofiltration was well below $60 \mathrm{~mL} / \mathrm{min} / 1.73 \mathrm{~m}^{2}$ for patients aged $\geq 60$ years, consistent with the view that both the upper and the lower limits of the normal range decrease with ageing. ${ }^{24}$

\section{Statistical analysis}

Data are expressed as mean $\pm \mathrm{SD}$ or median (IQR) for continuous variables, and number of cases and percentage for categorical variables. Comparisons among groups were performed by one-way analysis of variance or Kruskal-Wallis test, according to the parametric or nonparametric distribution of continuous variables, and by Pearson's $\chi^{2}$ test for categorical variables.

Crude mortality rates were described as events per 1000 patient-years, with 95\% exact Poisson CIs and adjusted for age and gender by a Poisson regression model. KaplanMeier survival probabilities for all-cause mortality were estimated according to the above categorizations and differences were analyzed using the log-rank statistic. The HRs and their 95\% CIs were estimated by Cox proportional hazards regression, using the 'normal eGFR' group (ie, the third decile and the G1b category) or the normofiltering group as reference. These analyses were adjusted for age and gender (model 1), plus albuminuria (model 2), plus CVD risk factors, that is, smoking, diabetes duration, HbAlc, BMI, triglycerides, total and HDL cholesterol, systolic and diastolic BP, and antihyperglycemic, lipid-lowering, antihypertensive, and renin-angiotensin system (RAS) blocker treatment (model 3), and plus other complications, that is, DR grade and any CVD, and presence of severe comorbidity(ies), that is, chronic obstructive pulmonary disease, chronic liver disease and/or cancer (model 4).

All $p$ values were two sided, and $p<0.05$ was considered statistically significant. Statistical analyses were performed using SPSS V.13.0 (SPSS, Chicago, IL, USA).

\section{RESULTS}

Valid information on vital status was retrieved for 15656 participants $(99.3 \%$ of the cohort). At the time of the census, $3602(23.0 \%)$ individuals had died; death rate was 31.0 per 1000 person-years (95\% CI 30.0 to 32.0 ) over a mean follow-up of $7.4 \pm 2.1$ years. ${ }^{25} 26$

\section{Clinical features of participants by eGFR deciles/KDIGO categories}

The clinical features of the RIACE participants stratified by eGFR deciles are shown in table 1 . From the first (highest) to the 10th (lowest) eGFR decile, the proportion of male patients and current smokers and the levels 


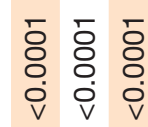

홍
홍

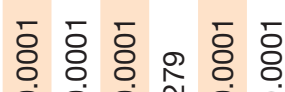

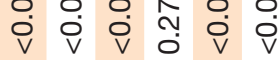

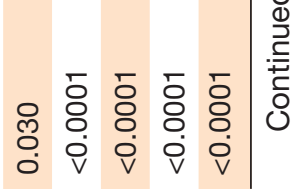

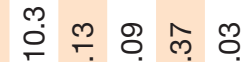

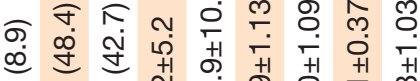

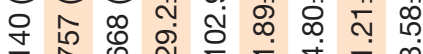

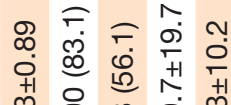
ก ก 0

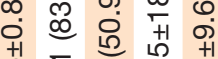
市

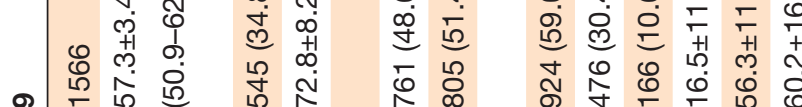

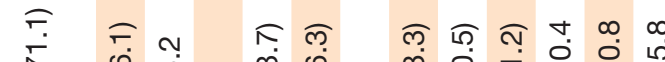
i

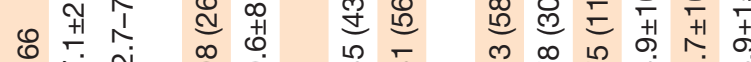

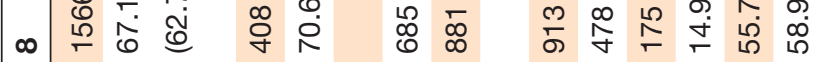
กิ ตุ

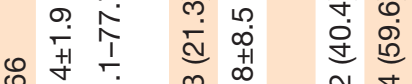

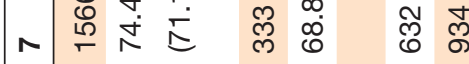

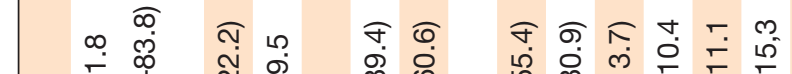

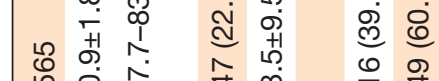

$\bullet$

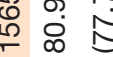

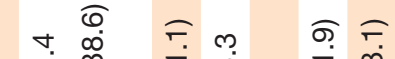

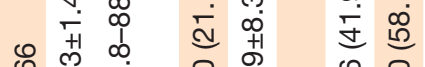

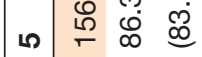

लำ

ஸึ

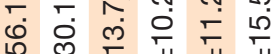

a $\sim$ ก

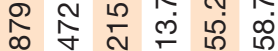

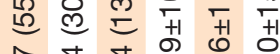

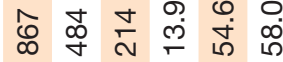

๑

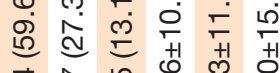

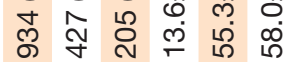

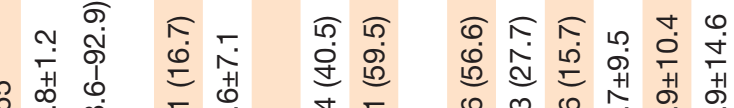

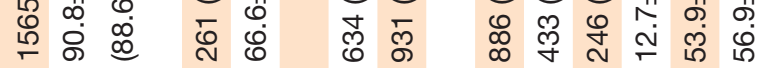

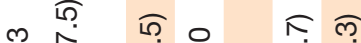

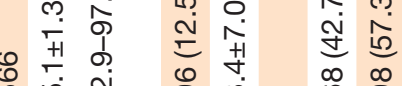

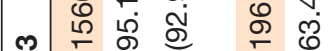

œ

กิ ชิ กุ

மำ

唄

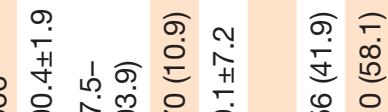

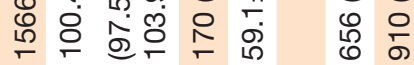

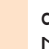

ก

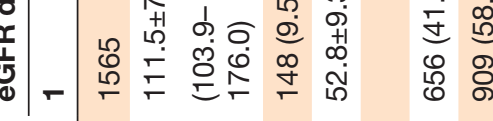

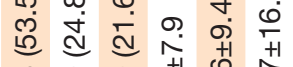
货

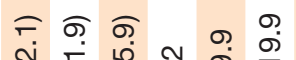
กิ

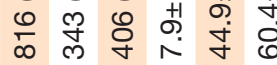

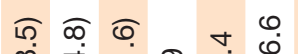

ธุ.

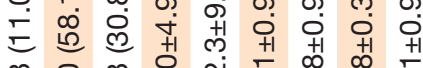


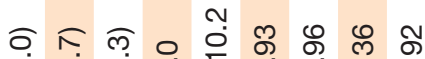

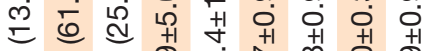

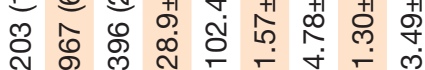
ช ¿ N

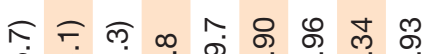
L 筞 เก

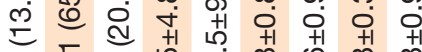

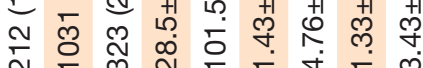
๙

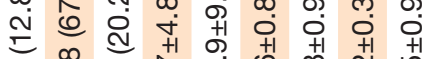

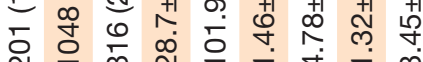

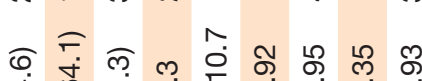
¿

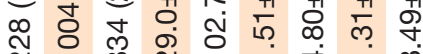
क ळ ○

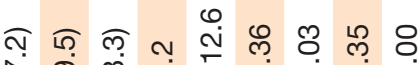
全 ơ

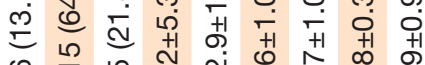

๓

苟 年 0ं ळ क्ष ก ヘิ

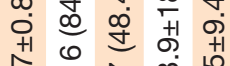
ลิ $\infty$

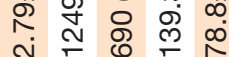
๗ ○ ก

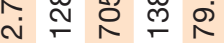
๙ ○ ल ம

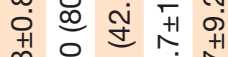
命 œ iो 5 ले ๗ ๓ ๓

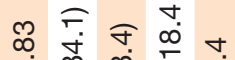
ơ

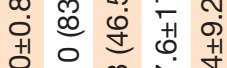

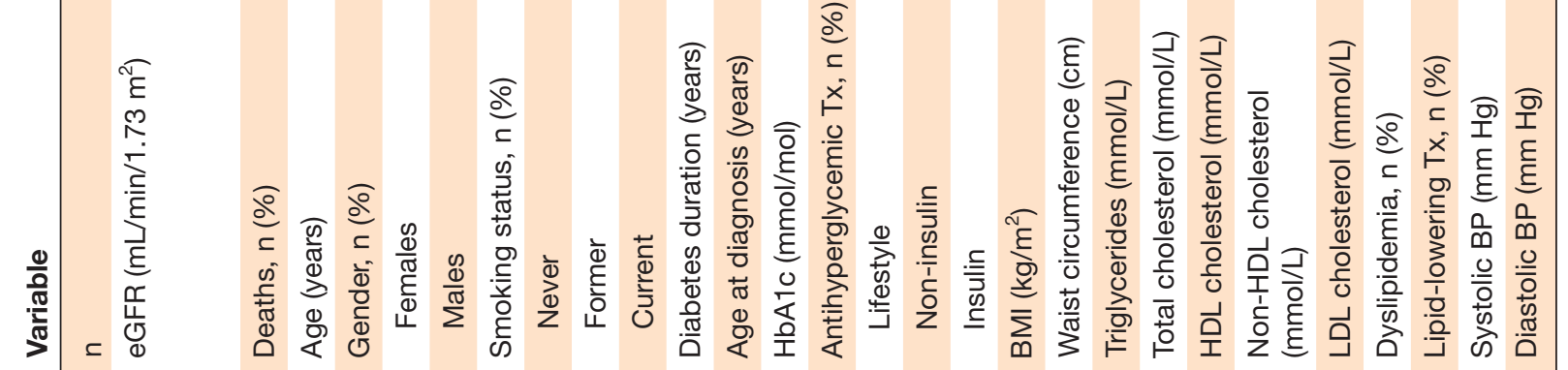




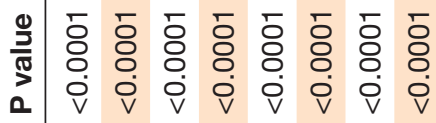

亏홓

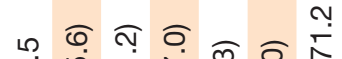

N

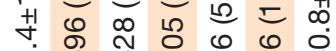

으 ๗ิ

๑

ஸ ฮ

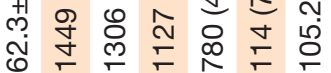

เก กุ

ก

卉

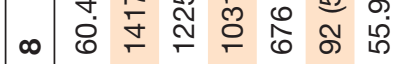

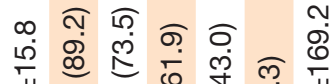

ने

$\wedge$ ช

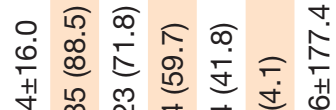

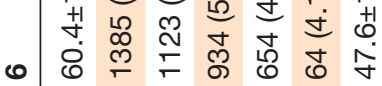

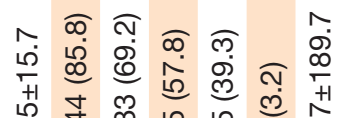

1) ำ

๓

ஸ்

ஸ்

$\checkmark$

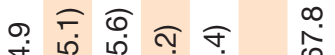

‡ ம்

๓

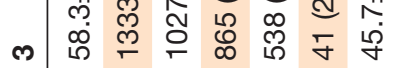

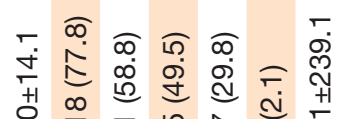

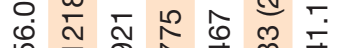

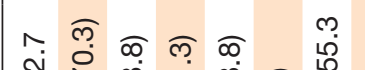

실

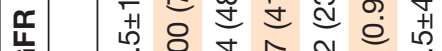

岁

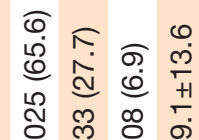

बे बें :

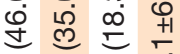

穴岱

लำ สิ

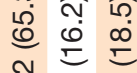

్ํ융

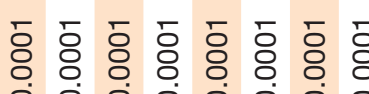

vi $\dot{v} \dot{v} \dot{v} \dot{v} \dot{v} \dot{v} \dot{v}$

চ

๖ั

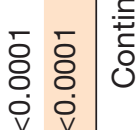

ชิ สุ สุ

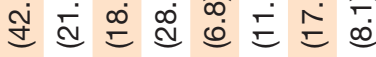

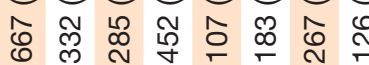

ติธ ส

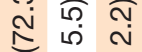

m $=$

$\stackrel{+}{\stackrel{4}{*}+\frac{1}{2}}$

लु

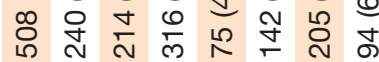

กิอ ชิ

芒

స

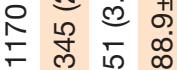

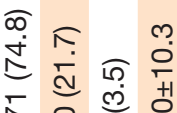

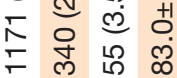

कุ लุ ตุ

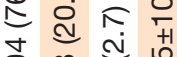

文

กิ

$\stackrel{n}{\stackrel{2}{*}} \stackrel{0}{=}$ กิ

กิ

하

$\stackrel{\infty}{\Sigma} \stackrel{\infty}{\infty}$ ○

D

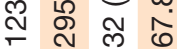

กิ

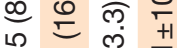

ก

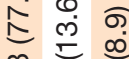

$\stackrel{m}{\frac{m}{n}}$ 운

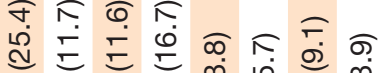

品

ণิ

$\stackrel{\infty}{E}$

空

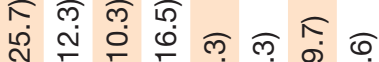

光

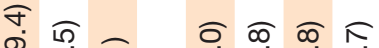

I $\stackrel{5}{\stackrel{2}{*}}$

๓ 0

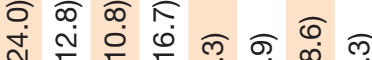

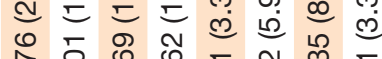

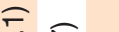

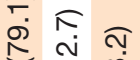

กิ ช $\overparen{ซ}$

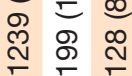

कุ

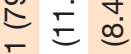

ำ ำ

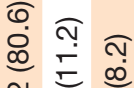

บิ $\underset{0}{\infty} \infty$

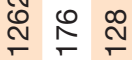

Ð

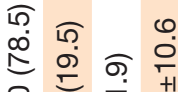

길

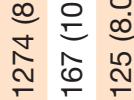

$\begin{array}{lll}\text { के } & & \\ \dot{1} & 0 & 0 \\ \infty & 0 & 0 \\ \infty & 0 & 0\end{array}$

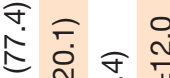

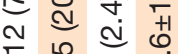

$\stackrel{\infty}{\infty}$ in

ต่ จ ลุ

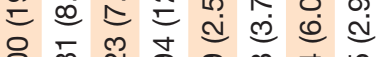

गு

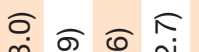

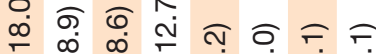

ऽ



$\widehat{\infty}-\widehat{\infty}$

๓

只

ริ ก

ம் กุ

N

న్

कุก

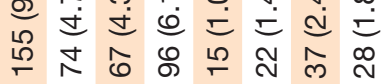

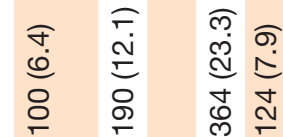

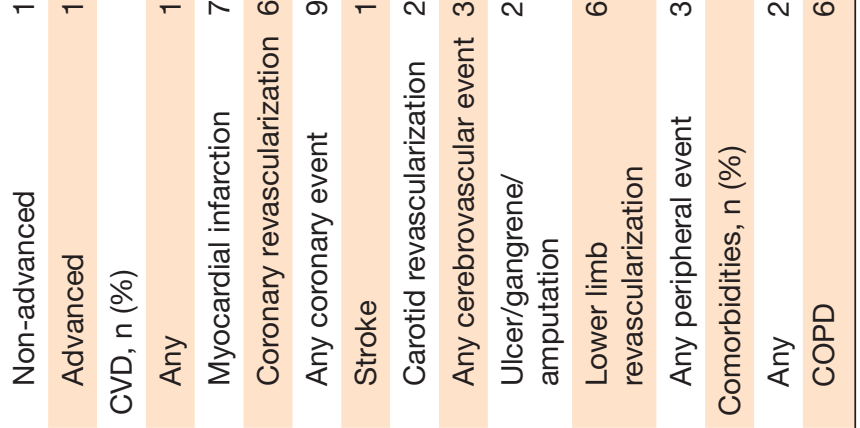

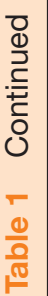

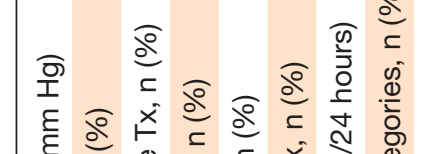

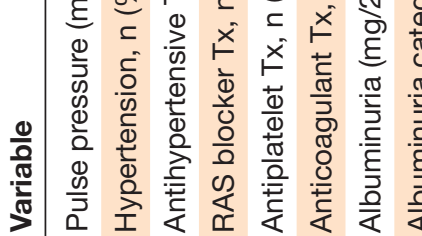
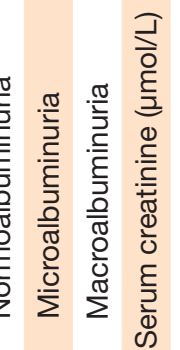

등 


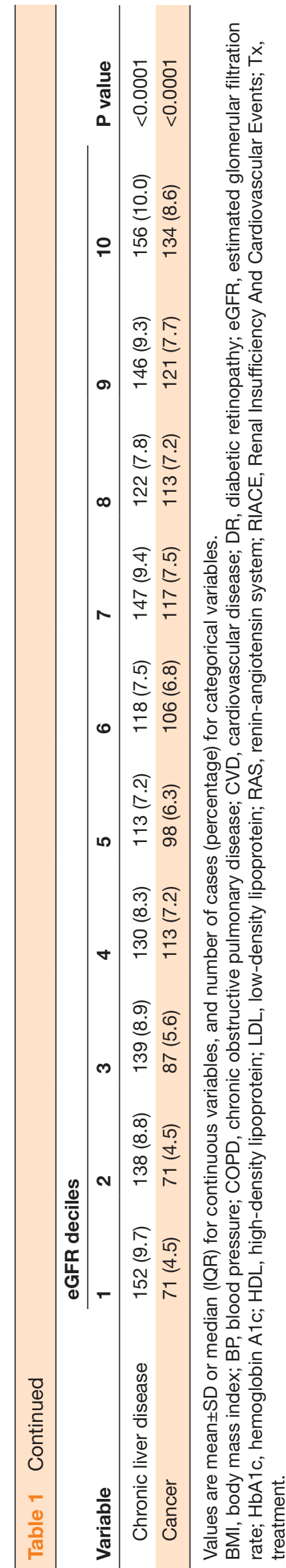

of LDL cholesterol and diastolic BP decreased, whereas age, age at diabetes diagnosis, diabetes duration, total cholesterol, systolic BP, pulse pressure, albuminuria, and prevalence of hypertension, dyslipidemia, DR, GVD, comorbidities, and insulin, lipid-lowering, antihypertensive, and RAS blocker treatment increased from the first to the tenth decile. Similar differences were observed among the KDIGO eGFR categories (online supplementary table S2).

\section{Association between eGFR deciles/KDIGO categories and mortality}

Crude mortality rates increased from the first to the tenth decile, with lower values in decile 1 and higher values in deciles 4-10, as compared with decile 3. However, when adjusted for age and gender, mortality rates were significantly higher in deciles 1,9 , and 10 compared with decile 3 (table 2). Kaplan-Meier estimates (online supplementary figure S2A) and unadjusted HRs (not shown) also increased from the first to the tenth decile. Again, when sequentially adjusting for confounders, mortality risk became significantly higher for decile 1 (HR 1.461 (95\% CI 1.175 to 1.818 ), $\mathrm{p}=0.001$ ) and remained significantly higher for deciles $9(1.312(95 \%$ CI 1.107 to $1.555), \mathrm{p}=0.002)$ and 10 (1.976 (95\% CI 1.673 to 2.333$)$, $\mathrm{p}<0.0001$ ), compared with decile 3 (figure 1 ). Results were similar when patients were stratified by KDIGO eGFR categories. In particular, when sequentially adjusting for confounders, mortality risk increased approximately to the same extent for G1a $(1.426(1.169-1.740), \mathrm{p}<0.0001)$ and G3a (1.332 (1.175-1.510), $\mathrm{p}<0.0001)$ and much more for G3b (1.963 (1.706-2.259), $\mathrm{p}<0.0001)$ and G4-5 (2.400 (2.001-2.878), $\mathrm{p}<0.0001)$ eGFR categories, compared with reference G1b category (online supplementary figure S3).

\section{Clinical features of participants by age (and gender-) corrected filtration status}

While individuals with an eGFR $>130 \mathrm{~mL} / \mathrm{min} / 1.73 \mathrm{~m}^{2}$ were only $44(0.3 \%)$, the number of hyperfiltering individuals rose to $644(4.1 \%)$ and $774(4.9 \%)$ using ageadjusted eGFR thresholds and age and gender-specific 95th and 5th percentiles, respectively, for defining hyperfiltration. Though the majority of these individuals $(57.9 \%$ and $66.9 \%$, respectively) fell in the first decile, there were also hyperfiltering patients falling in other deciles; conversely, the majority of individuals in the first decile were normofiltering $(76.2 \%$ and $66.9 \%$, respectively). Yet, the clinical features of hyperfiltering patients were similar to those of patients in the highest eGFR decile/category (table 3 and online supplementary table S3).

\section{Association between age (and gender-) corrected filtration} status and mortality

In hyperfiltering patients, unadjusted and age and genderadjusted mortality rates (table 2), Kaplan-Meier estimates (online supplementary figure S2B), and unadjusted 
Table 2 Mortality rates in the RIACE participants with valid information on vital status on 31 October 2015, stratified by eGFR deciles and filtration status

\begin{tabular}{|c|c|c|c|c|c|c|c|}
\hline & $\mathbf{n}$ & Events & $\begin{array}{l}\text { Percent } \\
\text { events }\end{array}$ & $\begin{array}{l}\text { Events per } 1000 \text { patient- } \\
\text { years }(95 \% \mathrm{Cl}) \text {, } \\
\text { unadjusted }\end{array}$ & $P$ value & $\begin{array}{l}\text { Events per } 1000 \text { patient- } \\
\text { years }(95 \% \mathrm{Cl}) \text {, age and } \\
\text { gender adjusted }\end{array}$ & $P$ value \\
\hline \multicolumn{8}{|c|}{ eGFR deciles } \\
\hline 1 & 1565 & 148 & 9.5 & 11.84 (10.08 to 13.92$)$ & 0.007 & 14.23 (11.68 to 17.33 ) & $<0.0001$ \\
\hline 2 & 1566 & 170 & 10.9 & 13.65 (11.74 to 15.86$)$ & 0.149 & 11.07 (9.18 to 13.35$)$ & 0.119 \\
\hline 3 & 1566 & 196 & 12.5 & 15.87 (13.80 to 18.26$)$ & Ref & 9.38 (7.85 to 11.21$)$ & Ref \\
\hline 4 & 1565 & 261 & 16.7 & 21.74 (19.25 to 24.54$)$ & 0.001 & 9.93 (8.41 to 11.73 ) & 0.544 \\
\hline 5 & 1566 & 330 & 21.1 & 28.17 (25.29 to 31.38$)$ & $<0.0001$ & 10.47 (8.93 to 12.27$)$ & 0.224 \\
\hline 6 & 1565 & 347 & 22.2 & 29.71 (26.74 to 33.00$)$ & $<0.0001$ & 10.66 (9.08 to 12.51$)$ & 0.154 \\
\hline 7 & 1566 & 333 & 21.3 & 28.35 (25.46 to 31.56$)$ & $<0.0001$ & 10.24 (8.70 to 12.04$)$ & 0.336 \\
\hline 8 & 1566 & 408 & 26.1 & 35.53 (32.25 to 39.15$)$ & $<0.0001$ & 11.57 (9.92 to 13.49$)$ & 0.016 \\
\hline 9 & 1566 & 545 & 34.8 & 50.32 (46.26 to 54.72$)$ & $<0.0001$ & 14.30 (12.35 to 16.55$)$ & $<0.0001$ \\
\hline 10 & 1565 & 864 & 55.2 & 92.51 (86.54 to 98.89 ) & $<0.0001$ & 23.66 (20.60 to 27.17 ) & $<0.0001$ \\
\hline \multicolumn{8}{|c|}{ Filtration status } \\
\hline Hyper & 644 & 169 & 26.2 & 36.32 (31.24 to 42.23$)$ & $<0.0001$ & 13.66 (11.30 to 16.51$)$ & 0.009 \\
\hline Normo & 12306 & 2160 & 17.6 & 22.93 (21.98 to 23.92$)$ & Ref & 10.81 (9.57 to 12.21$)$ & Ref \\
\hline Нуро & 2706 & 1273 & 47.0 & 73.86 (69.91 to 78.03$)$ & $<0.0001$ & 19.84 (17.42 to 22.59 ) & $<0.0001$ \\
\hline
\end{tabular}

eGFR, estimated glomerular filtration rate; RIACE, Renal Insufficiency And Cardiovascular Events.

(not shown) and adjusted HRs (figure 2) were all higher than in normofiltering and lower than in hypofiltering individuals. In model 4 , mortality risk was increased in hyperfiltering (1.231 (1.049-1.444), $\mathrm{p}=0.011)$ and much more in hypofiltering $(1.499(1.387-1.620), \mathrm{p}<0.0001)$ patients versus the normofiltering group. When using the age and gender-specific 95th and 5th percentiles, the extent of increase in mortality risk was similar for hyperfiltering individuals $(1.192(1.021-1.391), \mathrm{p}=0.026)$ and even more pronounced for hypofiltering patients $(2.125$

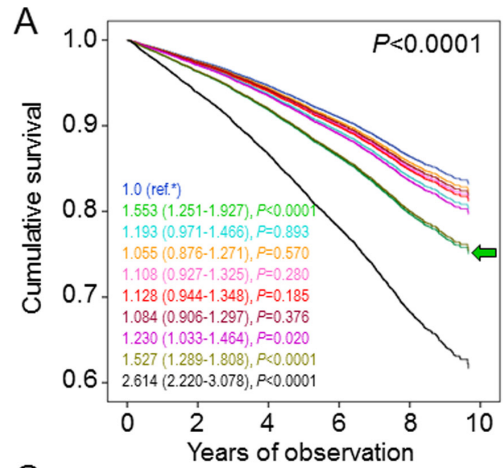

C

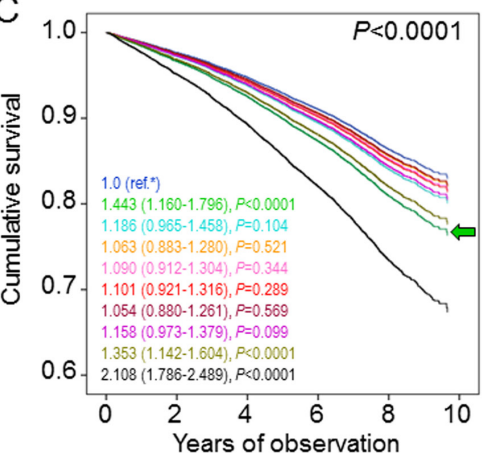

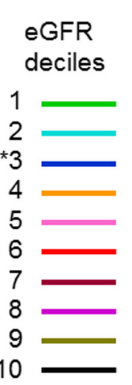
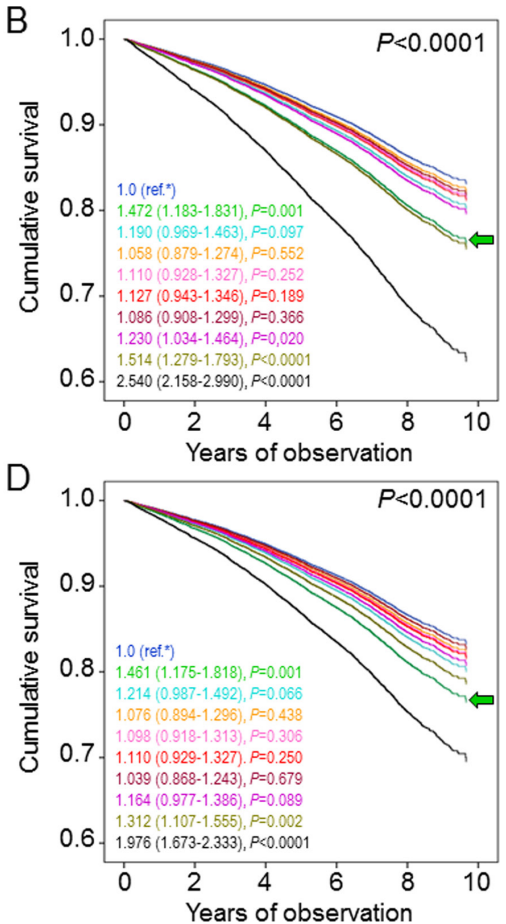

Figure 1 Cox proportional hazards regression adjusted for age and gender (A), plus albuminuria (B), plus CVD risk factors (C), plus complications/comorbidities (D), according to eGFR deciles. HRs (95\% Cl) for mortality are shown for each group; the green arrow indicates the first decile. CVD, cardiovascular disease; eGFR, estimated glomerular filtration rate. 
Table 3 Baseline clinical features in the RIACE participants with valid information on vital status on 31 October 2015, stratified by age-adjusted filtration status

\begin{tabular}{|c|c|c|c|c|}
\hline Variable & Normofiltering & Hyperfiltering & Hypofiltering & $P$ value \\
\hline n (\%) & 12306 (78.6) & $644(4.1)$ & $2706(17.3)$ & \\
\hline \multirow[t]{2}{*}{ eGFR $\left(\mathrm{mL} / \mathrm{min} / 1.73 \mathrm{~m}^{2}\right)$} & $86.3 \pm 13.6$ & $108.0 \pm 14.0$ & $46.0 \pm 11.2$ & \\
\hline & $(60.0-129.2)$ & $(74.5-176.0)$ & $(4.1-59.9)$ & \\
\hline Deaths, n (\%) & $2160(17.6)$ & $169(26.2)$ & $1273(47.0)$ & $<0.0001$ \\
\hline Age (years) & $64.9 \pm 9.9$ & $67.5 \pm 11.4$ & $73.8 \pm 8.6$ & $<0.0001$ \\
\hline Gender, n (\%) & & & & $<0.0001$ \\
\hline Females & $5107(41.5)$ & $294(45.7)$ & $1353(50.0)$ & \\
\hline Males & 7199 (58.5) & $350(54.3)$ & $1353(50.0)$ & \\
\hline Smoking status, n (\%) & & & & $<0.0001$ \\
\hline Never & $6891(56.0)$ & $368(57.1)$ & $1590(58.8)$ & \\
\hline Former & $3413(27.7)$ & $157(24.4)$ & $837(30.9)$ & \\
\hline Current & $2002(16.3)$ & 119 (18.5) & $279(10.3)$ & \\
\hline Diabetes duration (years) & $12.4 \pm 9.7$ & $11.9 \pm 9.8$ & $17.2 \pm 11.1$ & $<0.0001$ \\
\hline Age at diagnosis (years) & $52.6 \pm 11.0$ & $55.6 \pm 12.2$ & $56.6 \pm 12.0$ & $<0.0001$ \\
\hline $\mathrm{HbA} 1 \mathrm{c}(\mathrm{mmol} / \mathrm{mol})$ & $58.6 \pm 16.2$ & $59.6 \pm 18.8$ & $60.7 \pm 16.9$ & $<0.0001$ \\
\hline Antihyperglycemic Tx, n (\%) & & & & $<0.0001$ \\
\hline Lifestyle & $1767(14.4)$ & $93(14.4)$ & $253(9.3)$ & \\
\hline Non-insulin & 7791 (63.3) & 405 (62.9) & $1423(52.6)$ & \\
\hline Insulin & $2748(22.3)$ & $146(22.7)$ & $1030(38.1)$ & \\
\hline $\operatorname{BMI}\left(\mathrm{kg} / \mathrm{m}^{2}\right)$ & $29.0 \pm 5.1$ & $28.5 \pm 5.7$ & $29.1 \pm 5.1$ & 0.021 \\
\hline Waist circumference (cm) & $102.5 \pm 10.4$ & $101.4 \pm 11.6$ & $102.6 \pm 10.2$ & 0.029 \\
\hline Triglycerides (mmol/L) & $1.53 \pm 0.95$ & $1.51 \pm 1.41$ & $1.77 \pm 1.05$ & $<0.0001$ \\
\hline Total cholesterol (mmol/L) & $4.78 \pm 0.97$ & $4.77 \pm 1.05$ & $4.79 \pm 1.05$ & 0.957 \\
\hline HDL cholesterol (mmol/L) & $1.30 \pm 0.35$ & $1.36 \pm 0.37$ & $1.24 \pm 0.36$ & $<0.0001$ \\
\hline Non-HDL cholesterol (mmol/L) & $3.49 \pm 0.94$ & $3.41 \pm 1.02$ & $3.55 \pm 0.99$ & 0.001 \\
\hline LDL cholesterol (mmol/L) & $2.80 \pm 0.84$ & $2.74 \pm 0.84$ & $2.75 \pm 0.87$ & 0.012 \\
\hline Dyslipidemia, n (\%) & $10120(82.2)$ & $476(73.9)$ & $2260(83.5)$ & $<0.0001$ \\
\hline Lipid-lowering Tx, n (\%) & $5524(44.9)$ & $237(36.8)$ & $1477(54.6)$ & $<0.0001$ \\
\hline Systolic BP (mm Hg) & $137.8 \pm 17.8$ & $136.6 \pm 16.8$ & $139.7 \pm 19.2$ & $<0.0001$ \\
\hline Diastolic BP $(\mathrm{mm} \mathrm{Hg})$ & $79.1 \pm 9.3$ & $77.9 \pm 9.2$ & $77.5 \pm 9.9$ & $<0.0001$ \\
\hline Pulse pressure (mm Hg) & $58.7 \pm 15.4$ & $58.7 \pm 14.4$ & $62.2 \pm 17.1$ & $<0.0001$ \\
\hline Hypertension, n (\%) & $10378(84.3)$ & $521(80.9)$ & $2551(94.3)$ & $<0.0001$ \\
\hline Antihypertensive Tx, n (\%) & $8265(67.2)$ & $421(65.4)$ & $2386(88.2)$ & $<0.0001$ \\
\hline RAS blocker Tx, n (\%) & $6968(56.6)$ & $341(53.0)$ & $2031(75.1)$ & $<0.0001$ \\
\hline Antiplatelet Tx, n (\%) & 4507 (36.6) & $260(40.4)$ & $1481(54.7)$ & $<0.0001$ \\
\hline Anticoagulant Tx, n (\%) & $409(3.3)$ & $16(2.5)$ & $244(9.0)$ & $<0.0001$ \\
\hline Albuminuria (mg/24 hours) & $47.3 \pm 210.9$ & $77.0 \pm 678.7$ & $185.0 \pm 504.1$ & $<0.0001$ \\
\hline Albuminuria categories, $n(\%)$ & & & & $<0.0001$ \\
\hline Normoalbuminuria & $9532(77.5)$ & $452(70.2)$ & $1476(54.5)$ & \\
\hline Microalbuminuria & $2425(19.7)$ & $174(27.0)$ & $866(32.0)$ & \\
\hline Macroalbuminuria & $349(2.8)$ & $18(2.8)$ & $364(13.5)$ & \\
\hline Serum creatinine $(\mu \mathrm{mol} / \mathrm{L})$ & $73.0 \pm 14.9$ & $43.5 \pm 8.8$ & $127.0 \pm 55.6$ & $<0.0001$ \\
\hline $\mathrm{DR}, \mathrm{n}(\%)$ & & & & $<0.0001$ \\
\hline No & 9827 (79.9) & $518(80.4)$ & $1844(68.1)$ & \\
\hline
\end{tabular}

Continued 
Table 3 Continued

\begin{tabular}{|c|c|c|c|c|}
\hline Variable & Normofiltering & Hyperfiltering & Hypofiltering & $P$ value \\
\hline Non-advanced & $1440(11.7)$ & $72(11.7)$ & $435(16.1)$ & \\
\hline Advanced & $1039(8.4)$ & $54(8.4)$ & $427(15.8)$ & \\
\hline \multicolumn{5}{|l|}{ CVD, n (\%) } \\
\hline Any & 2453 (19.9) & $110(17.1)$ & $1057(39.1)$ & $<0.0001$ \\
\hline Myocardial infarction & $1173(9.5)$ & $51(7.9)$ & $518(19.1)$ & $<0.0001$ \\
\hline Coronary revascularization & 1090 (8.9) & $39(6.1)$ & $450(16.6)$ & $<0.0001$ \\
\hline Any coronary event & $1628(13.2)$ & $68(10.6)$ & $700(25.9)$ & $<0.0001$ \\
\hline Stroke & $336(2.7)$ & $18(2.8)$ & $159(5.9)$ & $<0.0001$ \\
\hline Carotid revascularization & $548(4.5)$ & $13(2.0)$ & $295(10.9)$ & $<0.0001$ \\
\hline Any cerebrovascular event & $841(6.8)$ & $28(4.3)$ & $423(15.6)$ & $<0.0001$ \\
\hline Ulcer/gangrene/amputation & $341(2.8)$ & $20(3.1)$ & $195(7.2)$ & $<0.0001$ \\
\hline Lower limb revascularization & $273(2.2)$ & $7(1.1)$ & $170(6.3)$ & $<0.0001$ \\
\hline Any peripheral event & $548(4.5)$ & $24(3.7)$ & $311(11.5)$ & $<0.0001$ \\
\hline \multicolumn{5}{|l|}{ Comorbidities, n (\%) } \\
\hline Any & $2053(16.7)$ & $130(20.2)$ & $604(22.3)$ & $<0.0001$ \\
\hline COPD & 435 (3.5) & $40(6.2)$ & $199(7.4)$ & $<0.0001$ \\
\hline Chronic liver disease & $1045(8.5)$ & $55(8.5)$ & $261(9.6)$ & 0.154 \\
\hline Cancer & 757 (6.2) & $53(8.2)$ & $221(8.2)$ & $<0.0001$ \\
\hline
\end{tabular}

Values are mean $\pm S D$ or median (IQR) for continuous variables, and number of cases (percentage) for categorical variables.

$\mathrm{BMI}$, body mass index; BP, blood pressure; COPD, chronic obstructive pulmonary disease; CVD, cardiovascular disease; DR, diabetic retinopathy; eGFR, estimated glomerular filtration rate; HbA1c, hemoglobin A1c; HDL, high-density lipoprotein; LDL, low-density lipoprotein; RAS, renin-angiotensin system; RIACE, Renal Insufficiency And Cardiovascular Events; Tx, treatment.
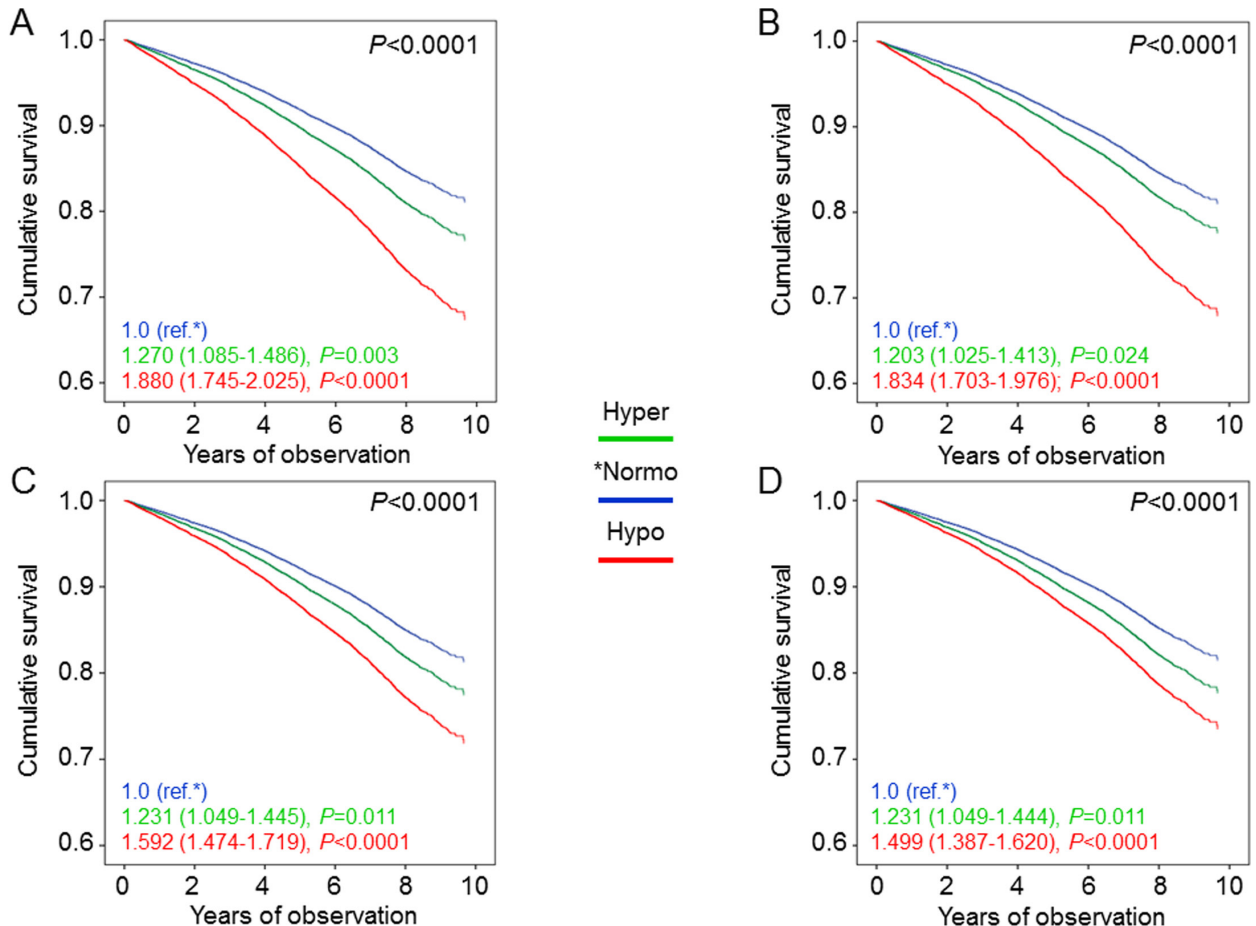

Figure 2 Cox proportional hazards regression adjusted for age and gender (A), plus albuminuria (B), plus CVD risk factors (C), plus complications/comorbidities (D), according to age-adjusted filtration status. HRs $(95 \% \mathrm{Cl})$ for mortality are shown for each group. CVD, cardiovascular disease; Hyper, hyperfiltering; Hypo, hypofiltering; Normo, normofiltering. 
(1.899-2.379), $\mathrm{p}<0.0001)$, consistent with the lower threshold for reduced eGFR levels (online supplementary figure $\mathrm{S} 4$ ).

\section{Association between other covariates and mortality}

All the other covariates included in the regression models were significantly associated with mortality, especially age (HR 1.09 per year), male gender (HR 1.33), current smoking (HR 1.25), insulin treatment (HR 1.84), history of major acute CVD events (HR 1.51), and severe comorbidity(ies) (HR 1.65). Diabetes duration, $\mathrm{BMI}$, systolic BP and, in some cases, triglycerides and RAS blocker treatment were excluded from the models.

\section{DISCUSSION}

This analysis of patients with type 2 diabetes from the RIACE cohort shows that 'renal hyperfiltration', regardless of how it is defined, is an independent predictor of subsequent death from any cause, thus suggesting that not only reduced, but also elevated eGFR may be associated with increased mortality in these individuals.

Previously, only two retrospective surveys focused exclusively on patients with type 2 diabetes and reported an increase in all-cause mortality among those in the highest eGFR category. ${ }^{27}{ }^{28}$ In particular, Luo $e t a l^{28}$ examined 4836 patients who had an acute stroke and found an increased risk of adverse stroke outcomes, including death, in those with an eGFR $\geq 120$ and $<45 \mathrm{~mL} /$ $\min / 1.73 \mathrm{~m}^{2}$, as compared with individuals with an eGFR of $90-119 \mathrm{~mL} / \mathrm{min} / 1.73 \mathrm{~m}^{2}{ }^{28}$ While these data refer to a specific subset of patients with type 2 diabetes (and were not adjusted for albuminuria), Davis et al studied 1296 individuals from the population-based Fremantle Diabetes Study and confirmed the J-shaped association between eGFR and mortality, with an increased risk for an eGFR $\geq 90$ and particularly $\geq 120 \mathrm{~mL} / \mathrm{min} / 1.73 \mathrm{~m}^{2}$, though the lowest risk was surprisingly observed among individuals with an eGFR of $45-59 \mathrm{~mL} / \mathrm{min} / 1.73 \mathrm{~m}^{2}{ }^{28}$ In the prospective, much larger RIACE study, risk of death was significantly increased for an eGFR below $\sim 60$ $\mathrm{mL} / \mathrm{min} / 1.73 \mathrm{~m}^{2}$ (deciles 9 and 10 or KDIGO categories G3-5) and above $\sim 105 \mathrm{~mL} / \mathrm{min} / 1.73 \mathrm{~m}^{2}$ (decile 1 or KDIGO category G1a), that is, starting from eGFR values in the high-normal range. Similar findings have been reported among 4201 adults with type 1 diabetes from the Finnish Diabetic Nephropathy Study. ${ }^{29}$ These data are consistent with previous large meta-analyses showing a J-shaped relationship between eGFR and allcause mortality in both non-diabetic and diabetic individuals. ${ }^{1011}$

However, the unique observation of our study is that an increased mortality risk was observed both in patients in the highest eGFR decile/category and in those above the age (and gender-) adjusted hyperfiltration thresholds, despite the fact that these two groups included different individuals, at least partly, thus raising the question whether the mechanisms involved are also different.
In fact, the detrimental effects of hyperfiltration on the kidney have been related to the increase in glomerular capillary hydraulic pressure that ensues when the increment in effective renal blood flow is lower than the increment in GFR, as indicated by an increased filtration fraction. ${ }^{1}$ Unfortunately, as no filtration fraction data were available, the role of glomerular hypertension could not be evaluated in the RIACE cohort. Indeed, it is possible either that patients with reduced renal mass had glomerular hypertension despite eGFR values in the highnormal range but below the hyperfiltration threshold or that hyperfiltering individuals had no increase in glomerular pressure, as occurs in living kidney donors, unless other factors superimpose ${ }^{30}$ and in normal pregnancy, in which both efferent and afferent arterioles dilate and the increase in renal blood flow is greater than the increase in GFR. ${ }^{31}$ However, despite including different individuals, the groups of participants with high-normal eGFR and hyperfiltration had quite similar clinical features, as in both cases patients were younger and more frequently male and current smoker and had a shorter diabetes duration and a lower prevalence of dyslipidemia, hypertension, complications and severe comorbidities, as compared with patients with low eGFR/hypofiltration (and even those with normal eGFR/normofiltration). Based on these features, individuals with high-normal eGFR/hyperfiltration were apparently not at higher risk of death compared with those falling in other eGFR categories or filtration groups, a finding that has important implications for interpreting the increased mortality risk associated with increasing eGFR values.

Another important finding of our study is that the increased risk of death associated with high-normal eGFR or hyperfiltration (ie, $\sim 1.5 \times$ and $\sim 1.2 \times$, respectively) remained virtually unchanged after adjustment for a wide range of confounders that may affect mortality and/or GFR estimation and was of approximately the same order of magnitude of the increase in mortality risk associated with some of these covariates, including prevalent CVD and comorbidities. Conversely, the increased mortality risk associated with low eGFR or hypofiltration was attenuated by sequential adjustment for confounders, because of the worse risk profile of these individuals, but remained higher than with high-normal eGFR (except for the ninth decile or the G3a category) or hyperfiltration, respectively. The unchanged and residual mortality risk associated with high-normal eGFR/hyperfiltration and low eGFR/hypofiltration, respectively, may be explained by unmeasured confounders or by the inability of 'statistical' adjustment to fully account for the impact of measured confounders. In addition, these findings raise a number of issues regarding the mechanisms underlying the relationship between high eGFR/hyperfiltration and mortality.

First, rather than the effect of supranormal renal function, with or without glomerular hypertension, this relationship may reflect the presence of confounders, which decrease serum creatinine levels, thus spuriously 
increasing eGFR. Low muscle mass is usually associated with conditions such as older age, malnourishing, cancer and other severe comorbidities, which confer an increased risk of death, and sarcopenia is in fact associated with increased mortality. ${ }^{32}$ The interpretation that the increased mortality associated with high eGFR is due to GFR overestimation because of muscle wasting in high-risk individuals is supported by the findings that the J-shaped association between creatininebased eGFR and death was observed only for all-cause mortality, ${ }^{10-1233}$ which includes death because of malignancies and other chronic conditions associated with cachexia, and disappeared in younger individuals. ${ }^{34}$ This view is further strengthened by the observation that the relationship between renal function and mortality was linear when using cystatin $\mathrm{C}, 3335$ which is not affected by muscle mass. ${ }^{37}$ However, the findings that high levels of measured GFR (by iohexol clearance) were also associated with subclinical CVD ${ }^{38}$ and that hyperfiltration was related to increased mortality independent of lean body (muscle) mass ${ }^{39}$ argue against this interpretation. In our study, the increased risk of death associated with highnormal eGFR/hyperfiltration remained after adjustment for age, gender, BMI, and severe comorbidities.

Second, the association between high eGFR/hyperfiltration and mortality may reflect early pathological changes occurring both in the kidney and in other vascular beds, possibly mediated by common mechanisms including RAS and sympathetic nervous system activation, which may be responsible for both hyperfiltration and premature death, with the former that may act either as a marker or a mediator of the increased mortality risk. ${ }^{13}$ This concept is supported by the association of high eGFR/hyperfiltration with GVD risk factors ${ }^{3840}$ subclinical CVD, ${ }^{223841}$ and CVD events. ${ }^{22} 274243$ However, high eGFR/hyperfiltration was not found to be associated with CVD mortality in most studies ${ }^{10-12} 3344$ and also the association with CVD events was not always observed. ${ }^{44}{ }^{45}$ Indeed, in the RIACE cohort, individuals with high eGFR/hyperfiltration had a lower, not a higher prevalence of CVD and overall CVD (and non-CVD) risk at baseline, as compared with patients in the other eGFR deciles/categories or filtration groups. Moreover, though they were more often current smokers, a condition associated with both high eGFR and mortality, the increased risk of death in individuals with high-normal eGFR/ hyperfiltration remained after adjustment for smoking status, consistent with a previous report. ${ }^{39}$

Third, the relationship between high eGFR/hyperfiltration and death may be mediated through the promotion of DKD, a powerful risk factor for CVD and all-cause mortality. ${ }^{46}$ This interpretation is consistent with the concurrent renal and CVD protection with RAS blockers and particularly with sodium-glucose cotransporter (SGLT2) inhibitors or glucagon-like peptide-1 (GLP-1) receptor agonists. ${ }^{47}$ In fact, hyperfiltration in diabetes has been related to increased sodium reabsorption due to the upregulation of SGLTs and sodium hydrogen exchanger-3, the latter modulated by GLP-1. ${ }^{3}$ The resulting decreased sodium delivery to the macula densa, which in obese individuals may derive also from compression of the thin loops of Henle, ${ }^{3}$ inhibits tubuloglomerular feedback, thereby reducing afferent arteriole resistance and increasing glomerular plasma flow and capillary hydraulic pressure. ${ }^{3}$ Moreover, imbalances in metabolic and neurohormonal factors, including RAS activation, act directly at the vascular level by reducing afferent more than efferent (or by increasing efferent) arteriole resistance. $^{3}$

The observed association between increased risk of death and high-normal eGFR/hyperfiltration, independent of confounders potentially affecting mortality and/ or GFR estimation, supports the concept that absolute or relative increases in eGFR might be considered a risk factor for all-cause mortality. However, further studies are needed for clarifying whether and how excess mortality is directly related to increased renal function. Furthermore, since our study was based on eGFR rather than on SNGFR, as all previous reports on the association between hyperfiltration and mortality, it was not possible to assess the role of glomerular hyperfiltration that, in individuals with reduced nephron endowment at birth or nephron loss after birth, may be associated also with normal or even reduced whole-kidney GFR.

A major strength of this study is that it analyzed exclusively patients with type 2 diabetes comparing different criteria for defining 'hyperfiltration'. Other strengths include the large sample size, the assessment of a wide range of clinical parameters, and the completeness of baseline and follow-up data. However, this study has several limitations. First, GFR estimation and lack of filtration fraction data and causes of death did not allow identifying individuals with renal hyperfiltration and those with glomerular hypertension and also evaluating the association of high eGFR/hyperfiltration with CVD mortality. However, measuring GFR (and renal plasma flow to calculate filtration fraction) is not feasible with such a large number of patients and, to date, only one study involving 1521 participants has used GFR measurements to evaluate the association of elevated GFR with CVD events. ${ }^{38}$ Furthermore, though CVD is responsible for most of the excess mortality associated with diabetes, also cancer and non-CVD/non-cancer deaths are more frequent in diabetic versus non-diabetic individuals ${ }^{48}$ and, as discussed above, all-cause, not CVD mortality has been consistently associated with 'hyperfiltration'. Second, lack of data on SNGFR (and birth weight, which may be associated with reduced nephron endowment and increased $\mathrm{SNGFR}^{49}$ ) did not allow to establish the role of glomerular hyperfiltration in the increased mortality risk. However, SNGFR measurements require invasive procedures that are not applicable to large observational studies. Third, in patients with diabetes with normal or elevated GFR, serum creatinine reflects renal function less accurately than cystatin $\mathrm{C},{ }^{50}$ which, however, is not routinely measured in these individuals. Fourth, lack of 
measurements of renal function over time did not allow assessing whether individuals with high eGFR/hyperfiltration developed more frequently renal disease during the follow-up. Fifth, lack of measurements of body composition did not allow to completely rule out the possibility of lower muscle mass in individuals with high-normal eGFR/hyperfiltration, though it was unlikely based on BMI and on the younger age and lower prevalence of severe comorbidities. Sixth, the study findings may not be applicable to the general ambulatory population, as only part of the individuals with type 2 diabetes attend diabetes clinics in Italy. Finally, the observational design makes causal interpretation impossible.

In conclusion, in individuals with type 2 diabetes, both high-normal eGFR and hyperfiltration are associated with an increased risk of death from any cause, independent of confounders that may directly impact on mortality and/or affect GFR estimation by reducing muscle mass. Further studies are required to establish whether the increased mortality risk is directly related to eGFR, either in the high-normal or the hyperfiltration range, and to clarify the mechanisms underlying this relationship.

\section{Author affiliations}

${ }^{1}$ Department of Clinical and Experimental Medicine, University of Pisa, Pisa, Italy

${ }^{2}$ Diabetes Service, Endocrinology Unit, IRCCS "Cà Granda - Ospedale Maggiore Policlinico" Foundation, Milan, Italy

${ }^{3}$ Department of Surgical, Medical, Molecular and Critical Area Pathology, University of Pisa, Pisa, Italy

${ }^{4}$ Division of Endocrinology, Diabetes and Metabolism, University and Hospital Trust of Verona, Verona, Italy

${ }^{5}$ Diabetes Unit, University of Siena, Siena, Italy

${ }^{6}$ Endocrinology and Diabetes Unit, Azienda Ospedaliera Papa Giovanni XXIII, Bergamo, Italy

${ }^{7}$ Department of Clinical and Experimental Medicine, University of Padua, Padua, Italy

${ }^{8}$ Department of Clinical and Biological Sciences, University of Turin, Orbassano, Italy

${ }^{9}$ Department of Internal Medicine, University of Turin, Turin, Italy

${ }^{10}$ Department of Emergency and Transplants, Section of Internal Medicine,

Endocrinology, Andrology and Metabolic Diseases, University of Bari Aldo Moro,

Bari, Italy

${ }^{11}$ Centre for Outcomes Research and Clinical Epidemiology (CORESEARCH), Pescara, Italy

${ }^{12}$ Department of Clinical and Molecular Medicine, "La Sapienza" University, Rome, Italy

Acknowledgements The authors thank the patients and the RIACE Investigators for participating in this study.

Contributors Study concept and design: GPe, EO, AS, and GPu. Data acquisition, analysis and interpretation: all authors. Drafting of the manuscript: GPu. Critical revision of the manuscript for important intellectual content: GPe, AS, EO, EB, CF, $\mathrm{RT}, \mathrm{MV}, \mathrm{FC}, \mathrm{GG}, \mathrm{LL}$, and AN. All authors read and approved the final version to be published.

Funding This research was supported by the Research Foundation of the Italian Diabetes Society (Diabete Ricerca) and the Diabetes, Endocrinology and Metabolism (DEM) Foundation, and by unconditional grants from Eli Lilly, Sigma-Tau, Takeda, Chiesi Farmaceutici, and Boehringer Ingelheim.

Competing interests GPe: lecture fees from AstraZeneca, Boehringer Ingelheim, Eli Lilly, Merck Sharp \& Dohme, Novo Nordisk, Sigma-Tau, and Takeda, and travel grants from AstraZeneca, Novo Nordisk, and Takeda. E0: consultant fees from Eli Lilly and Novo Nordisk. AS: consultant fees from AstraZeneca, Boehringer Ingelheim, and Sanofi-Aventis, and lecture fees from AstraZeneca, Boehringer Ingelheim, Eli Lilly, and Mundipharma. EB: consultant fees from Abbott, AstraZeneca, Boehringer Ingelheim, Bristol-Myers Squibb, Bruno Farmaceutici,
Eli Lilly, Janssen, Johnson \& Johnson, Merck Sharp \& Dohme, Mundipharma, Novartis, Novo Nordisk, Roche, Sanofi-Aventis, Servier, and Takeda, and research grants from AstraZeneca, Genzyme, Menarini Diagnostics, Novo Nordisk, Roche, and Takeda. CF: lecture fees from AstraZeneca, Boehringer Ingelheim, Eli Lilly, and Novo Nordisk and travel grants from AstraZeneca, Boehringer Ingelheim, Eli Lilly, Merck Sharp \& Dohme, Sanofi-Aventis, and Takeda. RT: consultant fees from AstraZeneca, Boehringer Ingelheim, Eli Lilly, Merck Sharp \& Dohme, Novo Nordisk, and Sanofi-Aventis, and lecture fees from AstraZeneca, Boehringer Ingelheim, Eli Lilly, and Novo Nordisk. MV: lecture fees from LifeScan and Novo Nordisk. FC: lecture fees from AstraZeneca, Sanofi-Aventis, and Takeda. GG: lecture fees from Boehringer Ingelheim and Mundipharma. LL: consultant fees from Abbott, AstraZeneca, Boehringer Ingelheim, Eli Lilly, Medtronic, Movi, Novo Nordisk, Roche, Sanofi-Aventis, and Takeda, and lecture fees from Abbott, AstraZeneca, Boehringer Ingelheim, Eli Lilly, Medtronic, Menarini, Merck Sharp \& Dohme, Movi, Mundipharma, Novo Nordisk, Roche, Sanofi-Aventis, and Takeda. AN: consultant fees from AstraZeneca, Pikdare, Roche, lecture fees from AstraZeneca, Boehringer Ingelheim, Medtronic, and Novo Nordisk, and research grants from Aboca, AstraZeneca, Eli Lilly, Novo Nordisk, Sanofi-Aventis, and Theras. GPu: consultant fees from AstraZeneca, Boehringer Ingelheim, and Eli Lilly, lecture fees from AstraZeneca, Boehringer Ingelheim, Eli Lilly, Merck Sharp \& Dohme, Mundipharma, Novartis, Novo Nordisk, Sigma-Tau, Takeda, and travel grants from AstraZeneca, Laboratori Guidotti, Sanofi-Aventis, and Takeda.

Patient consent for publication Not required.

Ethics approval The study was conducted in accordance with the Declaration of Helsinki. The research protocol was approved by the ethics committee of the coordinating center (Sant'Andrea Hospital, Rome, Italy) on 25 September 2006 (number 43/2006) and subsequently by the ethics committee of each participating center. Participants provided an informed consent.

Provenance and peer review Not commissioned; externally peer reviewed.

Data availability statement Data are available upon reasonable request. Deidentified participant data will be available upon reasonable request from the corresponding author, who will make a decision after consulting with the RIACE Steering Committee.

Open access This is an open access article distributed in accordance with the Creative Commons Attribution Non Commercial (CC BY-NC 4.0) license, which permits others to distribute, remix, adapt, build upon this work non-commercially, and license their derivative works on different terms, provided the original work is properly cited, appropriate credit is given, any changes made indicated, and the use is non-commercial. See: http://creativecommons.org/licenses/by-nc/4.0/.

ORCID iDs

Anna Solini http://orcid.org/0000-0002-7855-8253

Enzo Bonora http://orcid.org/0000-0003-1074-5164

Giuseppe Pugliese http://orcid.org/0000-0003-1574-0397

\section{REFERENCES}

1 Helal I, Fick-Brosnahan GM, Reed-Gitomer B, et al. Glomerular hyperfiltration: definitions, mechanisms and clinical implications. Nat Rev Nephrol 2012;8:293-300.

2 Cachat F, Combescure C, Cauderay M, et al. A systematic review of glomerular hyperfiltration assessment and definition in the medical literature. Clin J Am Soc Nephrol 2015;10:382-9.

3 Tonneijck L, Muskiet MHA, Smits MM, et al. Glomerular hyperfiltration in diabetes: mechanisms, clinical significance, and treatment. J Am Soc Nephrol 2017;28:1023-39.

4 Christiansen JS, Gammelgaard J, Frandsen M, et al. Increased kidney size, glomerular filtration rate and renal plasma flow in shortterm insulin-dependent diabetics. Diabetologia 1981;20:451-6.

5 Nelson RG, Bennett PH, Beck GJ, et al. Development and progression of renal disease in Pima Indians with non-insulindependent diabetes mellitus. N Engl J Med Overseas Ed 1996;335:1636-42.

6 Jerums G, Premaratne E, Panagiotopoulos S, et al. The clinical significance of hyperfiltration in diabetes. Diabetologia 2010;53:2093-104.

7 Gaspari F, Ruggenenti P, Porrini E, et al. The GFR and GFR decline cannot be accurately estimated in type 2 diabetics. Kidney Int 2013;84:164-73.

8 Pollak MR, Quaggin SE, Hoenig MP, et al. The glomerulus: the sphere of influence. Clin J Am Soc Nephrol 2014;9:1461-9.

9 Brenner BM, Lawler EV, Mackenzie HS. The hyperfiltration theory: a paradigm shift in nephrology. Kidney Int 1996;49:1774-7. 
10 Chronic Kidney Disease Prognosis Consortium, Matsushita K, van der Velde $\mathrm{M}$, et al. Association of estimated glomerular filtration rate and albuminuria with all-cause and cardiovascular mortality in general population cohorts: a collaborative meta-analysis. Lancet 2010;375:2073-81.

11 Fox CS, Matsushita K, Woodward M, et al. Associations of kidney disease measures with mortality and end-stage renal disease in individuals with and without diabetes: a meta-analysis. Lancet 2012;380:1662-73.

12 Mahmoodi BK, Matsushita K, Woodward M, et al. Associations of kidney disease measures with mortality and end-stage renal disease in individuals with and without hypertension: a meta-analysis. Lancet 2012;380:1649-61.

13 Kanbay M, Ertuglu LA, Afsar B, et al. Renal hyperfiltration defined by high estimated glomerular filtration rate: a risk factor for cardiovascular disease and mortality. Diabetes Obes Metab 2019;21:2368-83.

14 Penno G, Solini A, Bonora E, et al. Clinical significance of nonalbuminuric renal impairment in type 2 diabetes. $J$ Hypertens 2011;29:1802-9.

15 Orsi E, Solini A, Bonora E, et al. Haemoglobin A variability is a strong, independent predictor of all-cause mortality in patients with type 2 diabetes. Diabetes Obes Metab 2018;20:1885-93.

16 Penno G, Solini A, Bonora E, et al. Gender differences in cardiovascular disease risk factors, treatments and complications in patients with type 2 diabetes: the RIACE Italian multicentre study. $J$ Intern Med 2013;274:176-91.

17 Pugliese G, Solini A, Fondelli C, et al. Reproducibility of albuminuria in type 2 diabetic subjects. Findings from the renal insufficiency and cardiovascular events (RIACE) study. Nephrol Dial Transplant 2011;26:3950-4

18 Penno G, Solini A, Zoppini G, et al. Rate and determinants of association between advanced retinopathy and chronic kidney disease in patients with type 2 diabetes: the renal insufficiency and cardiovascular events (RIACE) Italian multicenter study. Diabetes Care 2012;35:2317-23.

19 Solini A, Penno G, Bonora E, et al. Diverging association of reduced glomerular filtration rate and albuminuria with coronary and noncoronary events in patients with type 2 diabetes: the renal insufficiency and cardiovascular events (RIACE) Italian multicenter study. Diabetes Care 2012;35:143-9.

20 Levey AS, de Jong PE, Coresh J, et al. The definition, classification, and prognosis of chronic kidney disease: a KDIGO controversies conference report. Kidney Int 2011;80:17-28.

21 Premaratne E, Macisaac RJ, Tsalamandris C, et al. Renal hyperfiltration in type 2 diabetes: effect of age-related decline in glomerular filtration rate. Diabetologia 2005;48:2486-93.

22 Reboldi G, Verdecchia P, Fiorucci G, et al. Glomerular hyperfiltration is a predictor of adverse cardiovascular outcomes. Kidney Int 2018:93:195-203

23 Yoo KD, Yoon H-J, Hwang S-S, et al. Different association between renal hyperfiltration and mortality by sex. Nephrology 2017:22:804-10.

24 Delanaye P, Schaeffner E, Ebert N, et al. Normal reference values for glomerular filtration rate: what do we really know? Nephrol Dial Transplant 2012;27:2664-72.

25 Penno G, Solini A, Orsi E, et al. Non-albuminuric renal impairment is a strong predictor of mortality in individuals with type 2 diabetes: the renal insufficiency and cardiovascular events (RIACE) Italian multicentre study. Diabetologia 2018;61:2277-89.

26 Penno G, Solini A, Bonora E, et al. Defining the contribution of chronic kidney disease to all-cause mortality in patients with type 2 diabetes: the renal insufficiency and cardiovascular events (RIACE) Italian multicenter study. Acta Diabetol 2018;55:603-12.

27 Luo $Y$, Wang $X$, Wang $Y$, et al. Association of glomerular filtration rate with outcomes of acute stroke in type 2 diabetic patients: results from the China national stroke Registry. Diabetes Care 2014;37:173-9.

28 Davis TME, Chubb SAP, Davis WA. The relationship between estimated glomerular filtration rate trajectory and all-cause mortality in type 2 diabetes: the Fremantle diabetes study. Eur J Endocrinol 2016;175:273-85.
29 Groop P-H, Thomas MC, Moran JL, et al. The presence and severity of chronic kidney disease predicts all-cause mortality in type 1 diabetes. Diabetes 2009;58:1651-8.

30 Grams ME, Sang Y, Levey AS, et al. Kidney-failure risk projection for the living kidney-donor candidate. N Engl J Med 2016;374:411-21.

31 Conrad KP, Jeyabalan A, Danielson LA, et al. Role of relaxin in maternal renal vasodilation of pregnancy. Ann N Y Acad Sci 2005;1041:147-54.

32 Oterdoom LH, Gansevoort RT, Schouten JP, et al. Urinary creatinine excretion, an indirect measure of muscle mass, is an independent predictor of cardiovascular disease and mortality in the general population. Atherosclerosis 2009;207:534-40.

33 Astor BC, Levey AS, Stevens LA, et al. Method of glomerular filtration rate estimation affects prediction of mortality risk. J Am Soc Nephrol 2009;20:2214-22.

34 Hallan SI, Matsushita K, Sang Y, et al. Age and association of kidney measures with mortality and end-stage renal disease. JAMA 2012;308:2349-60.

35 Shlipak MG, Sarnak MJ, Katz R, et al. Cystatin C and the risk of death and cardiovascular events among elderly persons. $N$ Engl J Med 2005;352:2049-60.

36 Reese PP, Feldman HI. More evidence that cystatin C predicts mortality better than creatinine. J Am Soc Nephrol 2009;20:2088-90.

37 Filler G, Bökenkamp A, Hofmann W, et al. Cystatin C as a marker of GFR--history, indications, and future research. Clin Biochem 2005;38:1-8.

38 Eriksen BO, Løchen M-L, Arntzen KA, et al. Subclinical cardiovascular disease is associated with a high glomerular filtration rate in the nondiabetic general population. Kidney Int 2014;86:146-53

39 Park M, Yoon E, Lim Y-H, et al. Renal hyperfiltration as a novel marker of all-cause mortality. J Am Soc Nephrol 2015;26:1426-33.

40 Tomaszewski M, Charchar FJ, Maric C, et al. Glomerular hyperfiltration: a new marker of metabolic risk. Kidney Int 2007;71:816-21.

41 Choi HM, Hyun YY, Lee KB, et al. High estimated glomerular filtration rate is associated with coronary artery calcification in middle-aged Korean men without chronic kidney disease. Nephrol Dial Transplant 2015;30:996-1001.

42 Di Angelantonio E, Chowdhury R, Sarwar N, et al. Chronic kidney disease and risk of major cardiovascular disease and non-vascular mortality: prospective population based cohort study. BMJ 2010;341:c4986.

43 Inrig JK, Gillespie BS, Patel UD, et al. Risk for cardiovascular outcomes among subjects with atherosclerotic cardiovascular disease and greater-than-normal estimated glomerular filtration rate. Clin J Am Soc Nephrol 2007;2:1215-22.

44 van der Sande NG, Blankestijn PJ, Leiner T, et al. High ratios of kidney function to kidney size are related to mortality and kidney function decline in high-risk patients. Eur J Prev Cardiol 2017;24:926-33.

45 Tonelli M, Klarenbach SW, Lloyd AM, et al. Higher estimated glomerular filtration rates may be associated with increased risk of adverse outcomes, especially with concomitant proteinuria. Kidney Int 2011;80:1306-14.

46 Gansevoort RT, Correa-Rotter R, Hemmelgarn BR, et al. Chronic kidney disease and cardiovascular risk: epidemiology, mechanisms, and prevention. Lancet 2013;382:339-52.

47 Zelniker TA, Wiviott SD, Raz l, et al. Comparison of the effects of glucagon-like peptide receptor agonists and sodium-glucose cotransporter 2 inhibitors for prevention of major adverse cardiovascular and renal outcomes in type 2 diabetes mellitus. Circulation 2019;139:2022-31.

48 Rao Kondapally Seshasai S, Kaptoge S, Thompson A, et al. Diabetes mellitus, fasting glucose, and risk of cause-specific death. N Engl J Med 2011;364:829-41.

49 Agarwal A. Single-nephron glomerular filtration rate in healthy adults. N Engl J Med 2017;377:1202.

50 Perkins BA, Nelson RG, Ostrander BEP, et al. Detection of renal function decline in patients with diabetes and normal or elevated GFR by serial measurements of serum cystatin C concentration: results of a 4-year follow-up study. J Am Soc Nephrol 2005;16:1404-12. 